\section{REDIMAT}

Journal of Research in Mathematics Education

\section{Hipatia Press}

www.hipatiapress.com

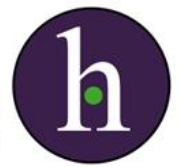

Instructions for authors, subscriptions and further details:

http://redimat.hipatiapress.com

\title{
Análisis de la Complejidad Cognitiva en la Lectura y Escritura de Expresiones Simbólicas Matemáticas
}

María Laura Distéfano ${ }^{1}$, Marcel David Pochulu ${ }^{2}, \&$ Vicenç Font ${ }^{3}$

1) Facultad de Ingeniería, Universidad de Mar del Plata, Argentina.

2) Instituto Académico Pedagógico de Ciencias Básicas y Aplicadas, Universidad Nacional de Villa María, Argentina.

3) Facultad de Educación, Universidad de Barcelona, España.

Date of publication: October $24^{\text {th }}, 2015$

Edition period: October 2015-February 2015

To cite this article: Distéfano, M.L, Pochulu, M.D., \& Font, V. (2015). Análisis de la complejidad cognitiva en la lectura y escritura de expresiones simbólicas matemáticas. REDIMAT, 4(3), 202-233. doi:

10.4471/redimat.2015.1568

To link this article: http://dx.doi.org/10.4471/redimat.2015.1568

\section{PLEASE SCROLL DOWN FOR ARTICLE}

The terms and conditions of use are related to the Open Journal System and to Creative Commons Attribution License (CC-BY). 


\section{Analysis of Cognitiv Complexity in Reading and Writing of Symbolic Mathematical Expressions}

Maria L. Distéfano

Universidad Nacional de Mar del Plata

Vicenç Font

Universitat de Barcelona
Marcel D. Pochulu

Universidad Nacional de

Villa María

(Received: 9 June 2015; Accepted: 4 October 2015; Published: 24 October 2015)

\section{Abstract}

This article focuses on an analysis of cognitive activities involved in reading and writing symbolic expressions, as part of the meaning process of mathematical symbols. Particularly, it has been centred on some symbols that are used exclusively in the mathematical field and whose use is required to freshmen of university careers that contain Mathematics in their curriculum. It was designed an ad hoc instrument to characterize the mathematical activity performed by using these symbols. An analysis of some items of the instrument is presented. It accounts for cognitive complexity that have the task of reading and writing symbolic expressions involving these particular symbols. To analyze the answers were used tools provided by the Ontosemiotic Approach (OSA). The combination of these tools reveals the multiplicity of processes involved in the tasks of reading and writing of symbolic expressions, as well as the network of necessary connections to give meaning, showing the cognitive complexity that the manipulation of mathematical symbols possesses.

Keywords: Symbolic expressions, configurations, semiotic functions 


\section{Análisis de la Complejidad Cognitiva en la Lectura y Escritura de Expresiones Simbólicas Matemáticas}

Maria L. Distéfano

Universidad Nacional de Mar del Plata
Marcel D. Pochulu

Universidad Nacional de

Villa María

Vicenç Font

Universitat de Barcelona

(Received: 9 June 2015; Accepted: 4 October 2015; Published: 24 October 2015)

\section{Abstract}

Este artículo está centrado en un análisis de las actividades cognitivas involucradas en la lectura y escritura de expresiones simbólicas, como parte del proceso de significación de símbolos matemáticos. Se analizan algunos símbolos que son de uso exclusivo en el ámbito matemático y a cuyo uso se enfrentan los estudiantes que ingresan a carreras universitarias que contienen Matemática en su plan de estudios. Para caracterizar la actividad matemática realizada al usar estos símbolos se diseñó un instrumento ad hoc. Se presenta un análisis sobre distintos ítems del instrumento que da cuenta de la complejidad cognitiva que poseen las tareas de lectura y escritura de expresiones simbólicas que involucran los símbolos en estudio. Para el análisis de las respuestas se utilizaron herramientas proporcionadas por el Enfoque Ontosemiótico (EOS). La combinación de estas herramientas pone de manifiesto la multiplicidad de procesos involucrados en las tareas de lectura y escritura de expresiones simbólicas, como así también la trama de vinculaciones necesarias para otorgar significado, evidenciando la complejidad cognitiva que la manipulación de símbolos matemáticos posee.

Keywords: Expresiones simbólicas, configuraciones, funciones semióticas 
a investigación en Didáctica de las Matemáticas ha puesto de manifiesto la importancia que tienen los aspectos semióticos en los proceso de enseñanza y aprendizaje de las matemáticas. La mirada semiótica a estos procesos - en particular gracias a los trabajos de Duval (2004) - ha planteado dos preguntas cruciales sobre el tipo de funcionamiento cognitivo que requiere la actividad y el pensamiento matemático: 1) ¿El funcionamiento del pensamiento matemático es (o no) independiente del lenguaje y de otros sistemas de representación semióticos utilizados?, y 2) ¿El pensamiento funciona en matemáticas de la misma manera (o no) que en otros dominios de conocimiento dadas las características específicas de los objetos matemáticos?

Se trata de dos preguntas abiertas con posicionamientos enfrentados entre los investigadores. Ahora bien, cualquier intento de responderlas ha de partir del hecho de que la actividad matemática se desarrolla en un contexto donde se usan signos ostensivos. Precisamente, la investigación que se presenta pretende ser una aportación al mejor conocimiento de la actividad matemática que se lleva a cabo al usar determinados signos matemáticos ostensivos. En particular, el interés está centrado en caracterizar la actividad matemática que realizan alumnos de los primeros cursos universitarios cuando responden tareas en las que han de usar los siguientes símbolos matemáticos: $\in, \subset, \forall, \exists, \wedge, \vee$. El avance en la caracterización de la actividad matemáticas realizada en este contexto, y en otros, puede dar alguna luz sobre la respuesta a las dos preguntas formuladas anteriormente.

Una de las razones que han llevado a seleccionar estos símbolos ha sido que los alumnos que ingresan a carreras universitarias que contienen Matemática en su plan de estudio, se enfrentan a la necesidad de leer y escribir utilizándolos, que son de uso exclusivo de esta ciencia y que no tienen sentido fuera de ella. Estos símbolos no son de uso frecuente en la escuela media pero son indispensables en el desarrollo de asignaturas de Matemática impartidas a nivel universitario. La habilidad del dominio de la escritura y lectura de expresiones que involucran este tipo de símbolos es necesaria para los estudiantes desde el momento inicial, ya que los docentes los emplean constantemente en el desarrollo de las clases, la bibliografía contiene gran cantidad de expresiones simbólicas en las que intervienen estos signos y se espera que ellos produzcan resoluciones que los requieren. Por otra parte, hay evidencias de dificultades en los alumnos para leer o 
escribir en forma simbólica utilizando dichos símbolos (Camós y Rodríguez; 2009; Distéfano, Urquijo y González, 2010; Lacués Apud, 2011).

Los signos matemáticos $\mathrm{y}$, más en general, las representaciones matemáticas no se pueden entender de manera aislada. Un símbolo lógico, una ecuación o una fórmula específica, una disposición concreta de bloques multibase, una gráfica particular en un sistema cartesiano, etc., adquieren sentido sólo como parte de un sistema más amplio con significados y convenciones que se han establecido. "Los sistemas representacionales importantes para las matemáticas y su aprendizaje tienen estructura, de manera que las diferentes representaciones dentro de un sistema están relacionadas de manera rica unas con otras" (Goldin y Stheingold, 2001, p. 2). Dentro de cada sistema representacional se incluyen las convenciones que lo configuran así como las relaciones con otros objetos y sistemas matemáticos. Algunos de los símbolos de uso frecuente en Matemática deben utilizarse e interpretarse incorporando las reglas de la Lógica que regulan su uso.

Para poder caracterizar la actividad matemática realizada al usar los símbolos $\in, \subset, \forall, \exists, \wedge, \vee$ como parte de un sistema más amplio con significados y convenciones establecidas para su uso, se diseñó un instrumento ad hoc. En este artículo se presenta un análisis sobre distintos ítems del instrumento que da cuenta de la complejidad cognitiva que poseen las tareas de lectura y escritura de expresiones simbólicas que involucran estos símbolos. Para el análisis de las respuestas se utilizó la metodología de análisis de la actividad matemáticas desarrollada por el Enfoque Ontosemiótico de la Cognición e Instrucción Matemática (EOS) (Godino, Batanero y Font, 2007, 2008). En particular se utilizaron las funciones semióticas y las configuraciones de objetos primarios. En el EOS, la noción de función semiótica se corresponde con la noción de representación, mientras que la configuración de objetos primarios y procesos (sistema semiótico) amplía a la de sistema de representación. El carácter reversible de la representación es también adoptado para la función semiótica: el antecedente y consecuente puede ser cualquier tipo de objeto primario (Godino y Font, 2010).

La estructura del artículo es la siguiente: a continuación de esta introducción, en la que se explican el problema y el objetivo de la investigación que se presenta, en el Capítulo 2 se expone el marco teórico 
utilizado (el EOS) y se hace una revisión de la literatura sobre el uso de los símbolos $\in, \subset, \forall, \exists, \wedge, \vee$. En el Capítulo 3 se detalla la metodología, sujetos participantes, características del cuestionario, etc. En el Capítulo 4 se explica el análisis de los datos realizado. El artículo termina con algunas consideraciones finales.

\section{Marco Teórico}

A continuación se presentan algunas nociones y constructos básicos del EOS, que han sido fundamento teórico en esta investigación.

En este enfoque, una práctica matemática, se define como cualquier acción, expresión o manifestación (lingüística o de otro tipo) realizada por alguien para resolver problemas matemáticos, comunicar la solución obtenida a otras personas, validar y generalizar esa solución a otros contextos (Godino, Batanero y Font, 2008). A partir de este concepto, surge la noción de significado. El significado se define entonces como "el sistema de prácticas operativas y discursivas para resolver un cierto tipo de problemas" (Godino, Bencomo, Font y Wilhelmi, 2007, p.7). Para el EOS, la cuestión del significado de los objetos matemáticos es de índole ontológica y epistemológica, puesto que se centra tanto en la naturaleza como en el origen de dichos objetos (Godino, Batanero y Font, 2008).

Para el EOS, el aprendizaje supone la apropiación por parte del estudiante de los significados validados en el seno de una institución, mediante su participación en las comunidades de prácticas (Godino, Bencomo, Font y Wilhelmi, 2007; Godino, Batanero y Font, 2008). Plantear el aprendizaje en términos de significados, otorga una relevancia central al proceso mediante el cual un sujeto crea un significado, vinculando una expresión con un contenido a través de una función semiótica. Esta función es establecida por un sujeto (persona o institución) de acuerdo con un cierto criterio o regla de correspondencia. De esta manera, los distintos objetos no resultan aislados entre sí, sino que se vinculan a través de las funciones semióticas construidas entre ellos. Dichos objetos pueden ejercer el rol de antecedente o de consecuente de la función semiótica (Godino Batanero y Font, 2008).

Debido al rol preponderante que juegan los objetos, el EOS considera que el problema epistémico-cognitivo no puede desligarse del ontológico. Así, la tipología de objetos primarios, u objetos de primer orden, según 
Font, Godino y Gallardo (2013), está constituida por: situacionesproblemas, elementos lingüísticos, conceptos, proposiciones y argumentos. Estas seis entidades primarias postuladas no son objetos aislados (Rondero y Font, 2015) sino que se vinculan entre sí: las situaciones-problemas son el origen y motivación de la actividad, el lenguaje actúa como soporte para representar a las restantes entidades y sirve de instrumento para la acción, los argumentos justifican los procedimientos y las proposiciones que, conjuntamente con las definiciones, resuelven las situaciones-problemas. Estas relaciones entre los objetos primarios determinan las configuraciones, definidas por Godino, Batanero y Font (2008) como "las redes de objetos intervinientes y emergentes de los sistemas de prácticas y las relaciones que se establecen entre los mismos." (p. 8). En la Figura 1 se esquematizan las relaciones que se establecen entre los objetos primarios en una configuración.
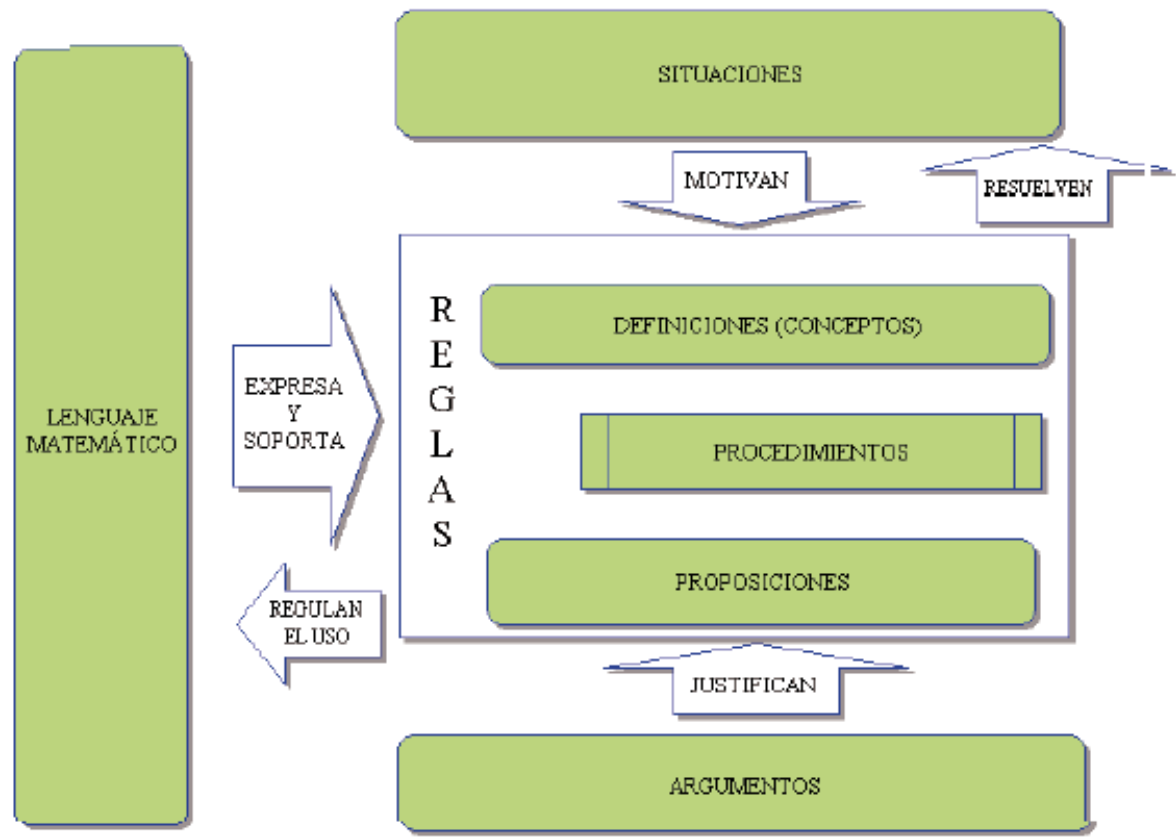

Figura 1. Relación de los objetos primarios en una configuración epistémica Fuente: Font y Godino (2006, p. 69) 
Los sistemas de prácticas y las configuraciones se proponen como herramientas teóricas para describir los conocimientos matemáticos, en su doble versión, personal e institucional. Por consiguiente, las configuraciones pueden ser epistémicas (redes de objetos institucionales) o cognitivas (redes de objetos personales).

Todos los elementos que conforman las configuraciones pueden ejercer el rol de expresión o contenido de funciones semióticas. De este modo, las funciones semióticas y la ontología matemática asociada tienen en cuenta la naturaleza relacional de la matemática y amplían el significado de representación.

\section{Revisión de la Literatura}

El uso de símbolos matemáticos y su incidencia en los procesos de enseñanza y aprendizaje han sido abordados por distintos autores, desde diversas líneas teóricas que abarcan perspectivas psicológicas, enfoques lingüísticos y aspectos didácticos (Kieran y Filloy, 1989; Pimm, 1990; Kaput, 1991; Rojano, 1994; Arcavi, 1994, 2007; Font, 2001). En todos los casos se destaca la relevancia del dominio del sistema simbólico matemático, su implicancia en la capacidad de resolver tareas problemáticas de un determinado nivel y en la posibilidad de expresar desarrollos y resultados.

En la mayoría de las publicaciones el interés está centrado en los primeros niveles de la educación formal (Hiebert, 1988; Booth, 1988; Gómez Granez, 1989; Socas y Palarea, 1997; Palarea Medina, 1999; Alcalá, 2002; Palencia y Talavera, 2004; Socas, 2007). Las temáticas abordadas están referidas al empleo de variables, a los símbolos de operaciones aritméticas, al uso del signo igual, a la resolución de ecuaciones y al manejo de expresiones algebraicas. También se enfocan en estos niveles de escolaridad las tesis doctorales de Palarea Medina (1998) y de Molina González (2006).

Focalizados en el nivel superior, las investigaciones de Camós y Rodríguez (2009) y de Colombano, Formica y Camós (2012), centran su investigación en la exploración y tipificación que los docentes hacen de los lenguajes natural y simbólico al enseñar los conceptos de límite, continuidad y derivabilidad, revelando como problemática la escasa atención que los docentes dan a la conversión entre registros y al uso simultáneo de ambos lenguajes. 
Por otra parte, Distéfano, Urquijo y González (2010), presentan una experiencia de enseñanza para mejorar las habilidades en el registro simbólico con estudiantes ingresantes a las carreras de Profesorado y Licenciatura en Matemática, y Lacués Apud (2011) refiere una experiencia de enseñanza de sistemas matemáticos de símbolos, con alumnos ingresantes a carreras de Ingeniería. En ambos casos se obtuvo una evaluación positiva de la intervención educativa y se concluye que es posible promover una mayor competencia en los estudiantes en relación con la utilización de símbolos matemáticos.

\section{Metodología}

Para el desarrollo de este trabajo, se ha considerado el modelo de análisis de la actividad matemática propuesto por el Enfoque Ontosemiótico del conocimiento y la instrucción matemática (Godino, Batanero y Font, 2007, 2008). Dicho modelo analiza la actividad matemática en términos de prácticas matemáticas y de configuraciones de objetos primarios activados en dichas prácticas:

1. Prácticas matemáticas. Descripción de las acciones realizadas para resolver las tareas matemáticas propuestas.

2. Configuraciones de objetos primarios y procesos matemáticos. Descripción de objetos y procesos matemáticos que intervienen en la realización de las prácticas, así como los que emergen de ellas. La finalidad de este nivel es describir la complejidad de los objetos y significados de las prácticas matemáticas. Tal complejidad es un elemento explicativo tanto de los conflictos de significado como de la progresión del aprendizaje.

Las características metodológicas de la investigación son de tipo interpretativo y cualitativo, ya que se pretende arribar a una comprensión profunda sobre las actividades llevadas a cabo por los estudiantes mediante un análisis inductivo/constructivo (Lincoln y Guba, 1985). El diseño metodológico escogido lleva a situar a la investigación en: Exploratoria (se pretende indagar sobre la construcción de significados, por parte de estudiantes universitarios, dentro del registro simbólico algebraico), Descriptiva (procurando caracterizar los rasgos fundamentales del proceso de significación de símbolos algebraicos); Etnográfica (se pretende comprender los acontecimientos tal y como los interpretan los sujetos investigados, a través de una inmersión en su pensamiento y práctica); Empírica (basada en la observación directa, con un trabajo fundamentado 
en hechos de experiencia directa no manipulados por la investigadora); De campo (la información es obtenida en el lugar de trabajo de los sujetos investigados); Hermenéutica (en el sentido de que se harán interpretaciones sobre las interpretaciones que hacen los estudiantes).

Se han considerado algunas variables cuantitativas (Marca como correcto, Marca incorrecto y corrige bien, Marca incorrecto y corrige mal, Sin resolver) asociadas a distintas categorías en la resolución, que complementan el análisis cualitativo pues permiten tener una noción del grado de resolución de cada ítem.

Para el estudio cualitativo, se utilizó la técnica de análisis denominada análisis semiótico (Godino, 2002), la cual permite describir de manera sistemática tanto la actividad matemática realizada al resolver problemas, como los objetos matemáticos primarios (elementos lingüísticos, conceptos/definiciones, proposiciones/propiedades, procedimientos y argumentos) que intervienen en las prácticas que permiten su resolución (Godino, Batanero y Font, 2007, 2008).

\section{Sujetos y Contexto}

El instrumento fue administrado a 101 estudiantes de primer año de las carreras de Ingeniería (Electrónica, Eléctrica, Electromecánica, Mecánica, Química, en Alimentos, en Materiales, Industrial), Bioquímica, Profesorado y Licenciatura en Matemática y Profesorado y Licenciatura en Biología, de la Universidad Nacional de Mar del Plata (Argentina). Los datos fueron relevados, en cada caso, a mediados del curso de Álgebra, durante el primer cuatrimestre de actividad académica.

\section{El Instrumento}

Para acotar la investigación, se hizo un recorte sobre los símbolos a estudiar, seleccionando seis símbolos de uso frecuente en los primeros cursos de asignaturas del área álgebra: $\in, \subset, \forall, \exists, \wedge, \vee$.

Se construyó un instrumento ad-hoc destinado a indagar en las prácticas que realizan los estudiantes en la lectura o en la formulación de expresiones simbólicas. En el proceso de diseño del mismo se elaboró una versión piloto y, luego de diversos ajustes, una versión final (ver Anexo 1), que consta de distintas tareas de lectura y escritura de expresiones simbólicas. 
La resolución de estas tareas requieren la escritura de ejemplos de uso, la constatación de estructuras sintácticas, conversiones entre los registros coloquial y simbólico-algebraico, y la asignación de valores de verdad.

Se trata de tareas con distinto nivel de complejidad: expresiones atómicas con símbolos de pertenencia o inclusión, expresiones moleculares con operador lógico de conjunción o disyunción y expresiones moleculares con una variable cuantificada. Esta variación en la complejidad de las expresiones estuvo destinada a que se pudiera extraer información de un modo segmentado.

Todas las expresiones refieren a unidades temáticas que se imparten en la escuela media, de modo que el contenido matemático al que la expresión refiere no debía resultar un obstáculo en la comprensión de la expresión.

\section{Las Funciones Semióticas Evaluadas en las Tareas}

A partir del análisis de la versión piloto del instrumento y de la exploración sobre las prácticas matemáticas que intervienen en la significación de los símbolos en estudio, se definieron las funciones semióticas principales que se consideraron como las asociadas a dichas prácticas (Distéfano, Aznar, Pochulu, 2014). De este modo, surgieron tres funciones semióticas que se definieron de la siguiente manera:

- F1: relaciona el símbolo con el vocablo de su denominación.

- F2: relaciona el vocablo /el símbolo con la estructura sintáctica de la expresión que lo contiene.

- F3: relaciona la proposición en la que está presente el símbolo, con su valor de verdad, el cual depende también de los significados de los restantes símbolos involucrados en la expresión.

A modo de ejemplo, se presentan en la Figura 2 estas funciones semióticas para el caso particular del símbolo de pertenencia, en el que se detallan el antecedente y el consecuente para cada una de ellas.

Con respecto a la función F2, es importante destacar que la sintaxis relativa al símbolo que aparece en el consecuente involucra tanto el orden de los elementos como los roles jugados por ellos. 


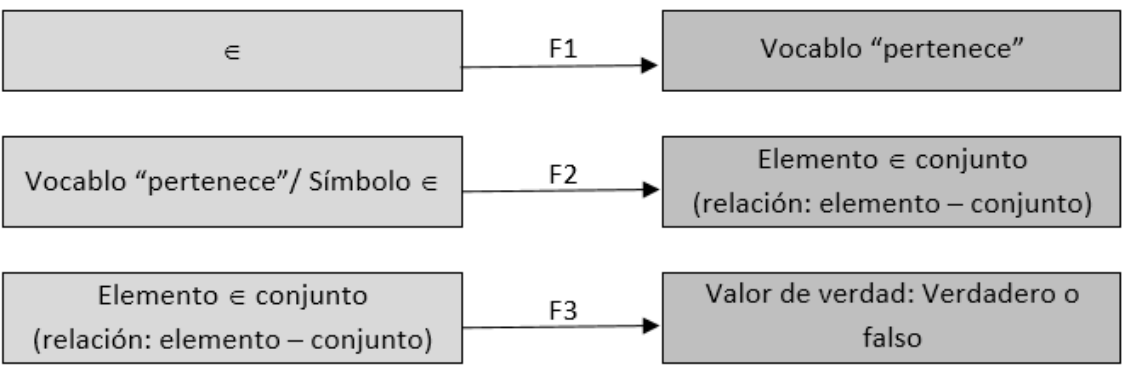

Figura 2. Funciones semióticas para el símbolo de pertenencia

En la tabla 1 se esquematizan las estructuras sintácticas correspondientes a los seis símbolos en estudio.

\section{Tabla 1}

Estructuras sintácticas correspondientes a los seis símbolos en el estudio

\begin{tabular}{|c|c|c|c|}
\hline $\begin{array}{l}\text { Estructura } \\
\text { sintáctica para } \\
\text { los símbolos } \\
\text { de pertenencia } \\
\text { y de inclusión }\end{array}$ & $\begin{array}{l}\text { Operando } \\
\text { Relacional de } \\
\text { la izquierda }\end{array}$ & $\begin{array}{l}\text { Relación } \\
\text { representada } \\
\text { por el símbolo }\end{array}$ & $\begin{array}{l}\text { Operando } \\
\text { Relacional de } \\
\text { la derecha }\end{array}$ \\
\hline $\begin{array}{l}\text { Estructura } \\
\text { sintáctica para } \\
\text { los símbolos } \\
\text { de conjunción } \\
\text { y de } \\
\text { disyunción }\end{array}$ & $\begin{array}{l}\text { Operando } \\
\text { Relacional de } \\
\text { la izquierda }\end{array}$ & $\begin{array}{l}\text { Operación } \\
\text { lógica } \\
\text { representada } \\
\text { por el símbolo }\end{array}$ & $\begin{array}{l}\text { Operando de la } \\
\text { derecha }\end{array}$ \\
\hline $\begin{array}{l}\text { Estructura } \\
\text { sintáctica para } \\
\text { los } \\
\text { cuantificadores }\end{array}$ & $\begin{array}{l}\text { Cuantificador } \\
\text { (universal o } \\
\text { existencial) }\end{array}$ & $\begin{array}{l}\text { Variable } \\
\text { cuantificada }\end{array}$ & $\begin{array}{l}\text { Esquema } \\
\text { proposicional }\end{array}$ \\
\hline
\end{tabular}

A continuación se presentan dos tareas incluidas en el instrumento (Ejercicio 1 y 2), que se han seleccionado para su discusión en este trabajo, 
puesto que dichas tareas ilustran el objetivo central del instrumento. Fueron diseñados para evaluar las funciones semióticas y sus enunciados se muestran en las Figuras 3 y 4, respectivamente.

1 Completar:
\begin{tabular}{|c|l|l|}
\hline Símbolo & ¿Cómo se lee? & $\begin{array}{l}\text { Escriba una expresión utilizando el } \\
\text { símbolo de la que se pueda afirmar } \\
\text { que es VERDADERA }\end{array}$ \\
\hline$\in$ & & \\
\hline$\subset$ & & \\
\hline$\forall$ & & \\
\hline$\exists$ & & \\
\hline$\wedge$ & & \\
\hline$\vee$ & & \\
\hline
\end{tabular}

Figura 3. Enunciado del Ejercicio 1 del instrumento

En el caso del Ejercicio 1, se evalúan las tres funciones semióticas vinculadas al significado de cada símbolo en estudio. La función semiótica F1 es evaluada en la respuesta de la primera columna, mientras que F2 y F3 son evaluadas en la resolución de la segunda columna, en una tarea de escritura.

El Ejercicio 2 fue diseñado para evaluar la función semiótica ligada a la sintaxis (F2) de cada símbolo, en una tarea de lectura. En los casos en los que la expresión dada no está correctamente escrita, la actividad es complementada con una tarea de escritura. Para cada uno de los símbolos se presenta una expresión correctamente escrita y una que no lo está, pero no aparecen en forma consecutiva para no inducir la respuesta.

\section{Resultados y Análisis}

Para analizar los resultados obtenidos, se construyó la configuración epistémica de cada ítem. Si bien en los análisis tradicionales en los que se emplean configuraciones como instrumento no se incluyen funciones semióticas, en este caso se las incorporó, de manera que la combinación de 
2 Determinar si las siguientes expresiones ESTÁN BIEN ESCRITAS. En caso de no estarlo re-escríbelas en forma correcta.

\begin{tabular}{|c|l|l|}
\hline Expresión & $\begin{array}{l}\text { Si la expresión está } \\
\text { BIEN ESCRITA señale } \\
\text { con una } \mathrm{x} \text { en esta } \\
\text { columna }\end{array}$ & $\begin{array}{l}\text { Si expresión está } \\
\text { MAL ESCRITA, re- } \\
\text { escribirla en forma } \\
\text { correcta en esta columna }\end{array}$ \\
\hline$-2 \in \mathbb{Z}$ & & \\
\hline $3 \subset \mathbb{Z}$ & & \\
\hline$\{1 ; 2\} \subset \mathbb{N}$ & & \\
\hline $\mathbb{N} \in \mathbb{Z}$ & & \\
\hline$[2 ; 5] \subset \mathbb{R}$ & & \\
\hline $4 \in \mathbb{N} \wedge-3<0$ & & \\
\hline$-1 \in \mathbb{N} \vee-1 \in \mathbb{Z}$ & & \\
\hline$-5 \wedge 4 \in \mathbb{R}$ & & \\
\hline $4 \in \mathbb{N} \vee \mathbb{Z}$ & & \\
\hline$\vee \mathbb{N} \quad \mathbb{N}>0$ & & \\
\hline$\vee x \in \mathbb{R} x^{2}>0$ & & \\
\hline$\exists x \in \mathbb{R} / y+2=5$ & &
\end{tabular}

Figura 4. Enunciado del Ejercicio 2 del instrumento

ambas herramientas permitiera un análisis más refinado de cada ítem, visualizando detalladamente las redes mediante las cuales se vinculan los elementos particulares de estas configuraciones de expresiones simbólicas.

La resolución de cada ítem involucra una gran cantidad de funciones semióticas, sin embargo se han incluido sólo aquellas que fueron definidas especialmente para esta investigación y algunas otras, a las que se denominó 'Auxiliares' (FA). Estas últimas no corresponden al proceso de significación propio del símbolo en estudio, pero necesariamente intervienen en la interpretación de la totalidad de la expresión. Por esta razón, en las figuras que siguen, se las representa con líneas punteadas y en color gris, mientras que las funciones semióticas principales son representadas en color negro y con líneas más gruesas. 


\section{Análisis del Ejercicio 1}

En la resolución del Ejercicio 1, claramente no hay una respuesta única al escribir un ejemplo.

Para construir la configuración epistémica de la resolución para el símbolo ' $\in$ ' se tomó un ejemplo tipo, que es ' $4 \in \mathrm{R}$ ', ya que la mayoría de los estudiantes respondió escribiendo algún número que pertenece al conjunto de los números reales.

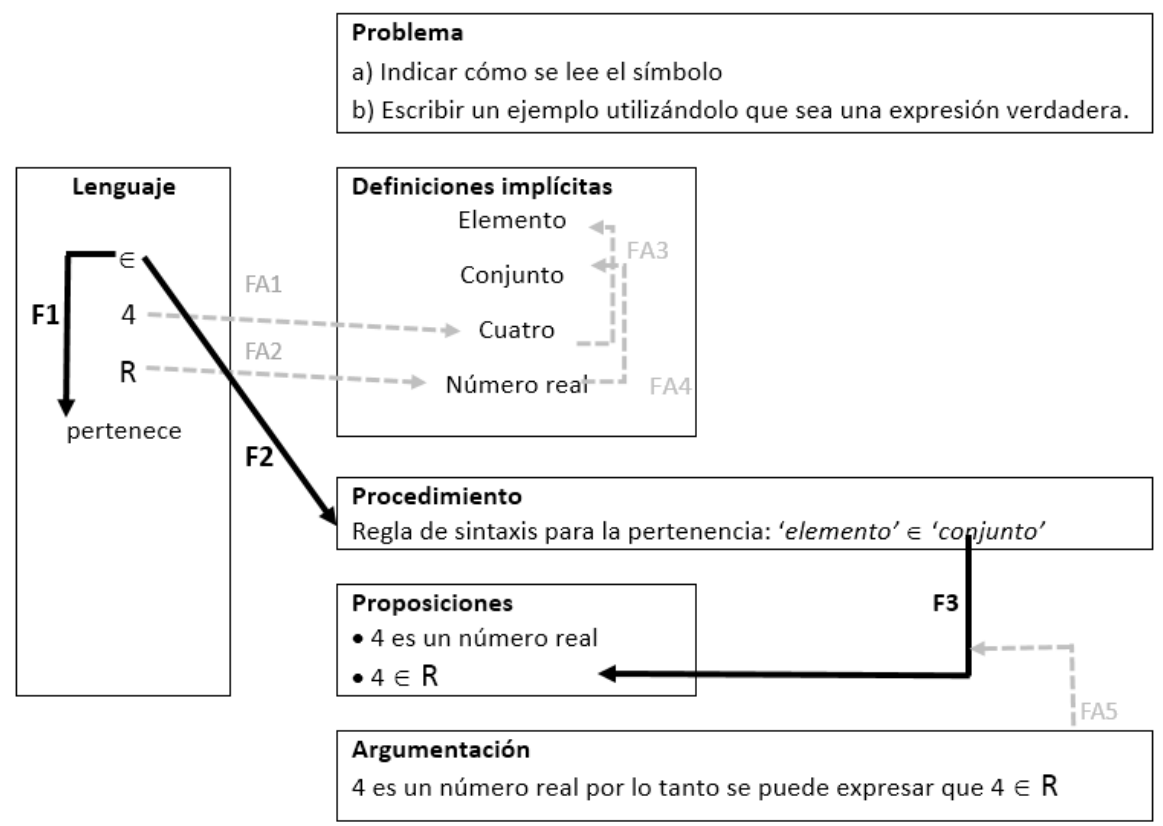

Figura 5. Configuración epistémica y funciones semióticas de la expresión $4 \in \mathbb{R}$ como ejemplo de una expresión que involucre al símbolo ' $\in$ '.

En la Figura 5 se presenta la configuración epistémica de la expresión ' $4 \in \mathrm{R}$ ' con algunas funciones semióticas incorporadas. Como se aclaró anteriormente, las flechas negras compactas representan las funciones semióticas definidas para la investigación, mientras que las grises punteadas representan a otras funciones semióticas que intervienen en el significado de la expresión, a las que se denominó 'Auxiliares'. 
La función semiótica $F 1$, que se establece entre el símbolo y su denominación coloquial, se manifiesta en la resolución de la primera columna, donde el estudiante debe completar 'Cómo se lee'.

En la resolución de la segunda columna del ejercicio, donde debe escribir un ejemplo utilizando el símbolo cuyo valor de verdad sea 'verdadero', se manifiestan las restantes funciones semióticas.

La función semiótica F2 se manifiesta en la precisión de la sintaxis empleada. En términos de los objetos de la configuración, liga un objeto del Lenguaje con el objeto Procedimiento. La función F3, asocia el Procedimiento con la Proposición que constituye la expresión formulada. Para su correcto establecimiento se requiere que conjuntamente intervenga la función auxiliar FA5, vinculando la Argumentación con la Proposición.

En la formulación de una expresión como ejemplo de uso del símbolo ' $\in$ ', algunos estudiantes escribieron expresiones del tipo ' $x \in R$ '. El uso de un literal (x) no permite analizar el establecimiento de la función F2, pues se debería asumir - aunque sin seguridad de ello - que dicho literal juega el rol de elemento. Los estudiantes que escribieron este tipo de expresiones, claramente no formularon Argumentaciones ni establecieron la función F3, pues no es posible determinar el valor de verdad.

En esta tarea el desempeño de los estudiantes es disímil para cada símbolo. En el caso del símbolo ' $\in$ ', todos los alumnos lo conocen y 90 de ellos escriben un ejemplo con un correcto valor de verdad. Sólo 56 estudiantes saben cómo se lee el símbolo ' $C$ ' y 46 de ellos muestran un ejemplo con un correcto valor de verdad. En el caso de los cuantificadores, todos los estudiantes manifiestan saber cómo se lee pero sólo la mitad aproximadamente escribe un ejemplo utilizándolo que tenga valor de verdad Verdadero.

Para los símbolos ' $\wedge$ ' y ' $\vee$ ', 90 estudiantes saben cómo se lee, pero sólo un tercio de ellos, aproximadamente, muestra un ejemplo correcto.

\section{Análisis del Ejercicio 2}

La resolución del Ejercicio 2 requiere analizar si una serie de expresiones está correctamente escrita $y$, en caso de no estarlo, reescribirla correctamente. Es decir que este ejercicio está dirigido a evaluar aspectos sintácticos ligados a los símbolos en estudio, tanto en la tarea de lectura 
como en la de escritura. Considerando que para cada uno de los seis símbolos se incluyó una expresión correctamente escrita como una que no lo está, para cada uno de ellos se está evaluando ambos tipos de tareas.

Las resoluciones de los ítems que tienen expresiones correctamente escritas determinaron tres categorías disjuntas:

- Marca Como Correcto: El estudiante manifestaría tener establecida la F2 del símbolo principal de la expresión, pues resuelve correctamente la tarea de lectura.

- Marca Incorrecto y Corrige: El estudiante manifestaría no tener establecida la F2 del símbolo pues comete errores en la lectura y en la escritura.

- Sin Resolver: No se puede realizar afirmaciones.

En la Tabla 2 se presenta la cantidad de alumnos en cada una de las categorías. Puede observarse que, en general, estos ítems fueron correctamente resueltos. La mayor cantidad de errores se produjeron en los que aparece el símbolo de disyunción y de inclusión.

Tabla 2

Alumnos en cada categoría de los ítems con expresiones correctamente escritas

\begin{tabular}{cccc}
\hline Categoría & $\begin{array}{c}\text { Marca } \\
\text { como } \\
\text { correcto }\end{array}$ & $\begin{array}{c}\text { Marca } \\
\text { incorrecto } \\
\text { corrige }\end{array}$ & Sin resolver \\
Ítem & 96 & 5 & 0 \\
$\{1 ; 2\} \subset \mathbb{N}$ & 67 & 27 & 7 \\
$\{2 ; 5\} \subset \mathbb{R}$ & 62 & 32 & 7 \\
$4 \in \mathbb{N} \wedge-3<0$ & 89 & 7 & 5 \\
$-1 \in \mathbb{N} \vee-1 \in \mathbb{Z}$ & 54 & 45 & 2 \\
$\forall x \in \mathbb{R} x^{2}>0$ & 71 & 29 & 1 \\
$\exists x \in \mathbb{Z} / x<0$ & 92 & 8 & 1 \\
\hline
\end{tabular}

Por su parte, los ítems en los que la expresión está incorrectamente escrita fijaron cinco categorías disjuntas: 
- Marca Incorrecto y Corrige Bien: Manifiestan tener establecida la F2 del símbolo pues resuelven correctamente tanto la tarea de lectura como la de escritura.

- Marca Incorrecto pero Corrige Mal: Si bien en la lectura parecieran tener construida la F2, el corregir mal indicaría que no es así, por lo tanto puede considerarse que no tienen establecida la F2

- Marca Incorrecto pero No Corrige: Probablemente la respuesta fue azarosa

- Marca Como Correcto: Manifestarían no tener establecida la F2 del símbolo en una tarea de lectura.

- Sin Resolver: No se puede realizar afirmaciones.

En la Tabla 3 se presentan las cantidades de alumnos en cada categoría. En casi todos estos ítems son muy pocos los estudiantes que resuelven bien, es decir que deciden que la expresión está incorrectamente escrita y la reescriben correctamente.

Tabla 3

Alumnos en cada categoría de los items con expresiones incorrectamente escritas

\begin{tabular}{|c|c|c|c|c|c|}
\hline Categoría & $\begin{array}{c}\text { Marca } \\
\text { incorrect } \\
\text { o y } \\
\text { corrige } \\
\text { bien }\end{array}$ & $\begin{array}{c}\text { Marca } \\
\text { incorrect } \\
\text { o pero } \\
\text { corrige } \\
\text { mal }\end{array}$ & $\begin{array}{c}\text { Marca } \\
\text { incorrect } \\
\text { o pero } \\
\text { no } \\
\text { corrige }\end{array}$ & $\begin{array}{c}\text { Marca } \\
\text { como } \\
\text { correcto }\end{array}$ & $\begin{array}{c}\text { Sin } \\
\text { resolver }\end{array}$ \\
\hline $3 \subset \mathbb{Z}$ & 26 & 22 & 3 & 42 & 7 \\
\hline $\mathbb{N} \in \mathbb{Z}$ & 29 & 14 & 2 & 56 & 0 \\
\hline$-5 \wedge 4 \in \mathbb{R}$ & 7 & 18 & 0 & 74 & 2 \\
\hline $4 \in \mathbb{N} \vee \mathbb{Z}$ & 17 & 45 & 2 & 35 & 2 \\
\hline$\forall \mathbb{N} \quad \mathbb{N}>0$ & 18 & 20 & 1 & 58 & 4 \\
\hline$\exists x \mathbb{R} / y+2=5$ & 62 & 3 & 0 & 34 & 2 \\
\hline
\end{tabular}

Como puede observarse a partir de los datos de las Tablas anteriores, los ítems de este ejercicio que presentaron mayores dificultades a los estudiantes son los que corresponden a las expresiones que están 


\section{Distéfano, Pochulu and Font - Expresiones simbólicas}

incorrectamente escritas. Las dificultades se manifestaron tanto en la tarea de lectura (las consideran correctamente escritas) como en la tarea de escritura (las reconocen como incorrectamente escitas pero las corrigen mal).

A continuación, en las Figuras 6 a 10 se presentan las configuraciones correspondientes a algunos de los ítems En dichas configuraciones se consideraron los objetos intervinientes tanto la actividad de lectura como la de escritura. También se incorporaron las funciones semióticas que involucradas en el esquema de cada configuración, tanto las definidas en relación al significado del símbolo en estudio como las denominadas 'auxiliares' que vinculan otros objetos que son relevantes al análisis.

\section{Configuración del ítem $3 \subset \mathbb{Z}$}

En este ítem, la función semiótica F2 del símbolo de inclusión interviene en la tarea de lectura, para decidir que la expresión no está correctamente escrita.

Dado que la expresión puede ser reescrita de dos formas $(3 \in Z$ ó $\{3\}$ $\subset \mathrm{Z}$ ), en la tarea de escritura puede intervenir la función semiótica F2 del símbolo ' $\in$ 'o la del símbolo ' $C$ '.

En los casos en los que reescriben mal, puede considerarse que la F2 del ' $\subset$ ' no está establecida a pesar de que el alumno haya decidido que no está correctamente escrita.

Las funciones semióticas auxiliares FA1 y FA2 vinculan los símbolos que intervienen en la expresión con su correspondiente definición en lenguaje coloquial, mientras que FA3 y FA4 relacionan la definición asociada al símbolo con la categoría general a la que pertenece. Esto incidirá en la decisión de la regla de sintaxis a utilizar, ya sea para definir si la expresión está correctamente escrita como para reescribirla. Finalmente, FA5 y FA6 intervienen, desde la Argumentación, en la decisión sobre la corrección o no de la regla de sintaxis utilizada $(\mathrm{F} 2)$, respectivamente en la lectura y en la escritura.

La gran cantidad de estudiantes que considera la expresión como bien escrita (42) podría provenir de la confusión que se genera en torno a la similitud semántica que las expresiones "pertenecer" y "estar incluido" tienen en el lenguaje coloquial. La construcción deficiente del significado 
de cada uno de los símbolos ' $\in$ ' y ' $C$ ' hace que los estudiantes no adviertan la precisión que tiene su uso en el quehacer matemático.

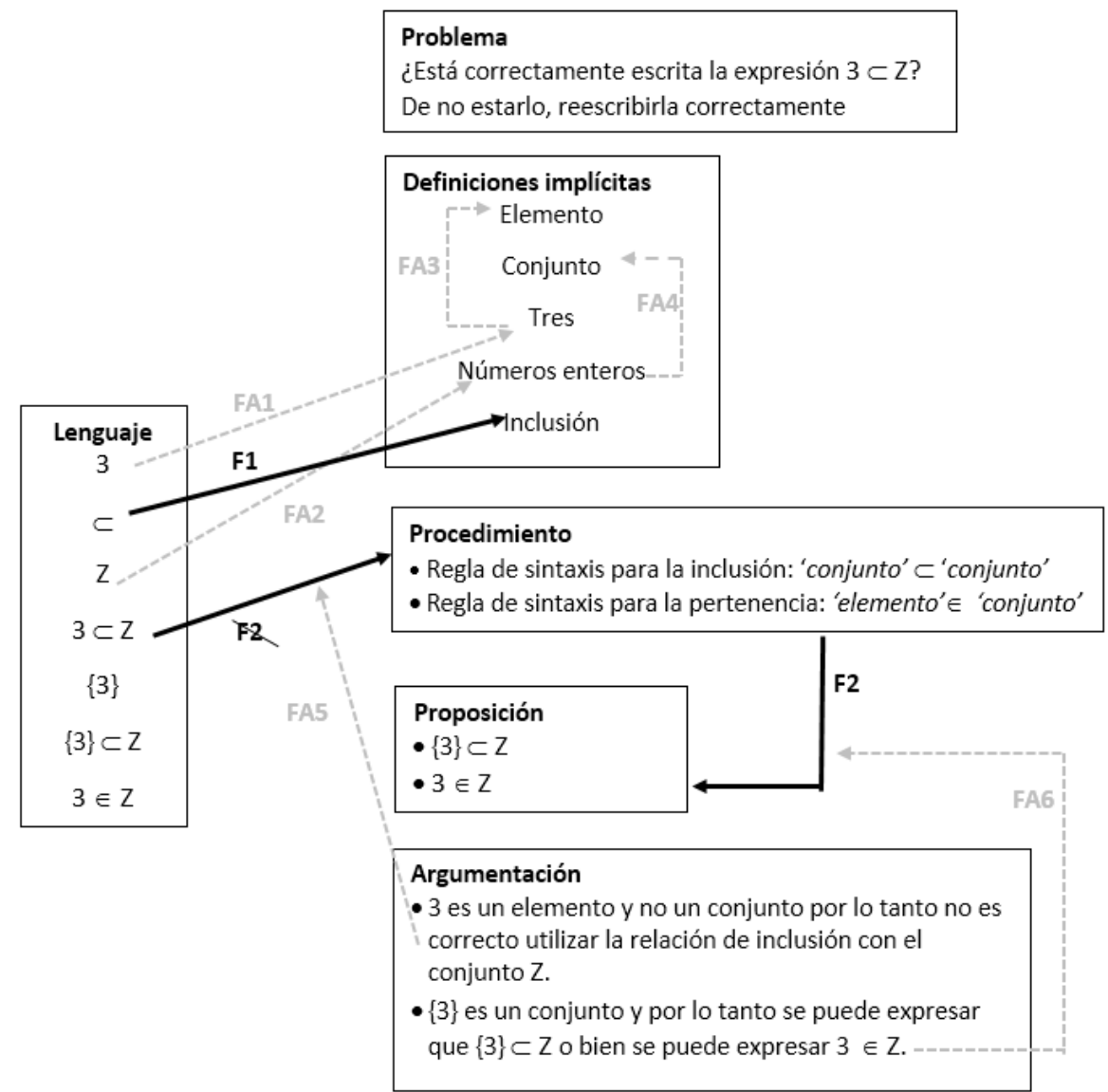

Figura 6. Configuración epistémica y funciones semióticas del análisis de correcta escritura de la expresión $3 \subset \mathbb{Z}$

* F2 indica que la función semiótica F2 está involucrada en tanto que el alumno la necesita para determinar que no se verifica la regla de sintaxis.

Considerando el hecho de que sólo 26 estudiantes resolvieron correctamente este ítem reescribiendo la expresión apropiadamente, 
conduce a pensar que el nivel de construcción del significado del símbolo de inclusión es muy bajo.

\section{Configuración del ítem $\mathbb{N} \in \mathbb{Z}$}

En este ítem intervienen dos funciones semióticas relativas a la sintaxis (F2), la del símbolo ' $\in$ ' para determinar que la expresión no está correctamente escrita y la del símbolo ' $C$ ' para reescribir la expresión.

Como en el caso anterior, intervienen funciones semióticas auxiliares, unas que relacionan los restantes símbolos que constituyen la expresión y otras que inciden en la decisión de la incorrecta escritura como en la reformulación de la expresión.

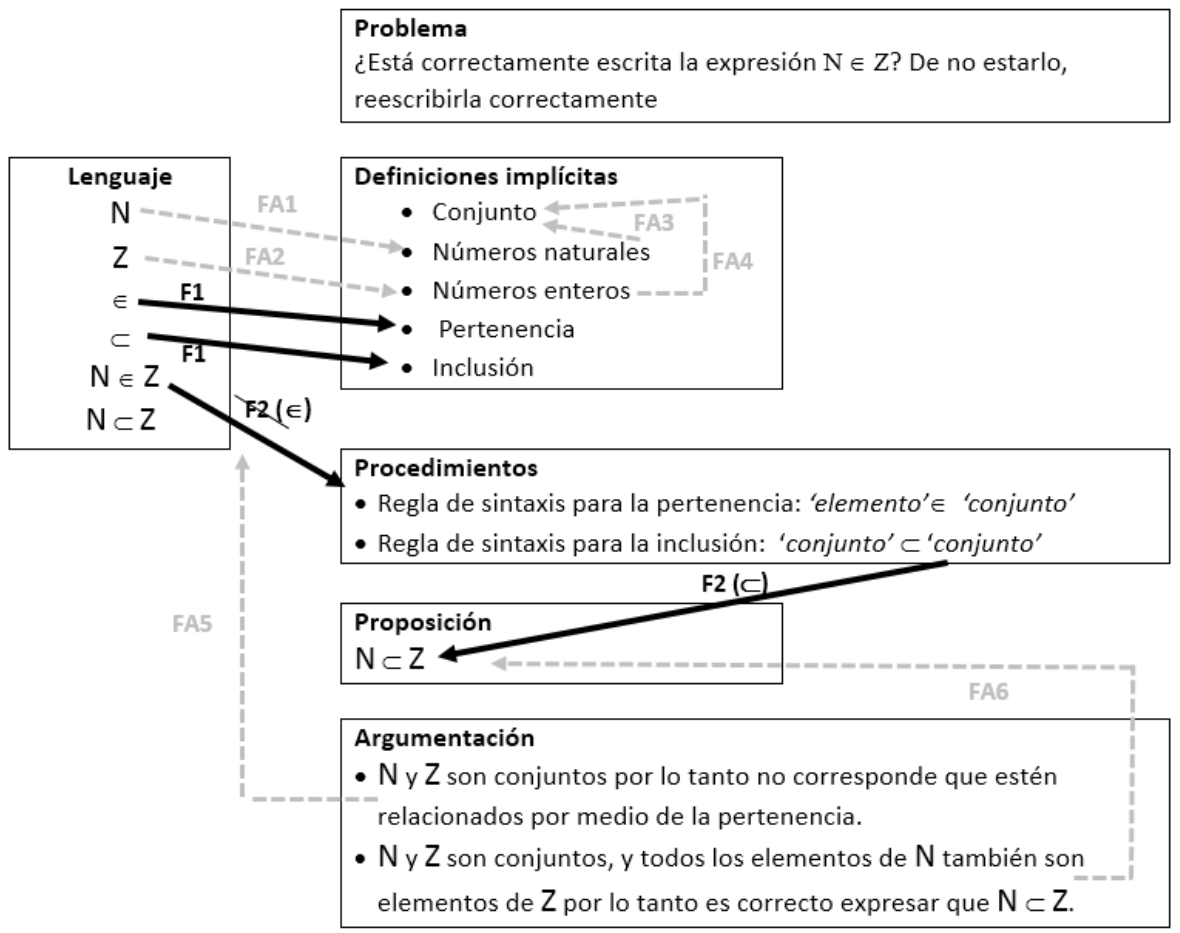

Figura 7. Configuración epistémica y funciones semióticas del análisis de correcta escritura de la expresión $\mathbb{N} \in \mathbb{Z}$ 
También en este caso la baja cantidad de alumnos que resuelven correctamente podría adjudicarse a la confusión coloquial entre las expresiones "pertenecer" y "estar incluido", que incide sobre la construcción del significado de los símbolos ' $\in$ ' y ' $\subset$ '.

\section{Configuración del ítem $-1 \in \mathbb{N} \vee-1 \in \mathbb{Z}$}

En este ítem la expresión está correctamente escrita, por lo tanto no debe hacerse ninguna modificación en ella para obtener una expresión sintácticamente adecuada.

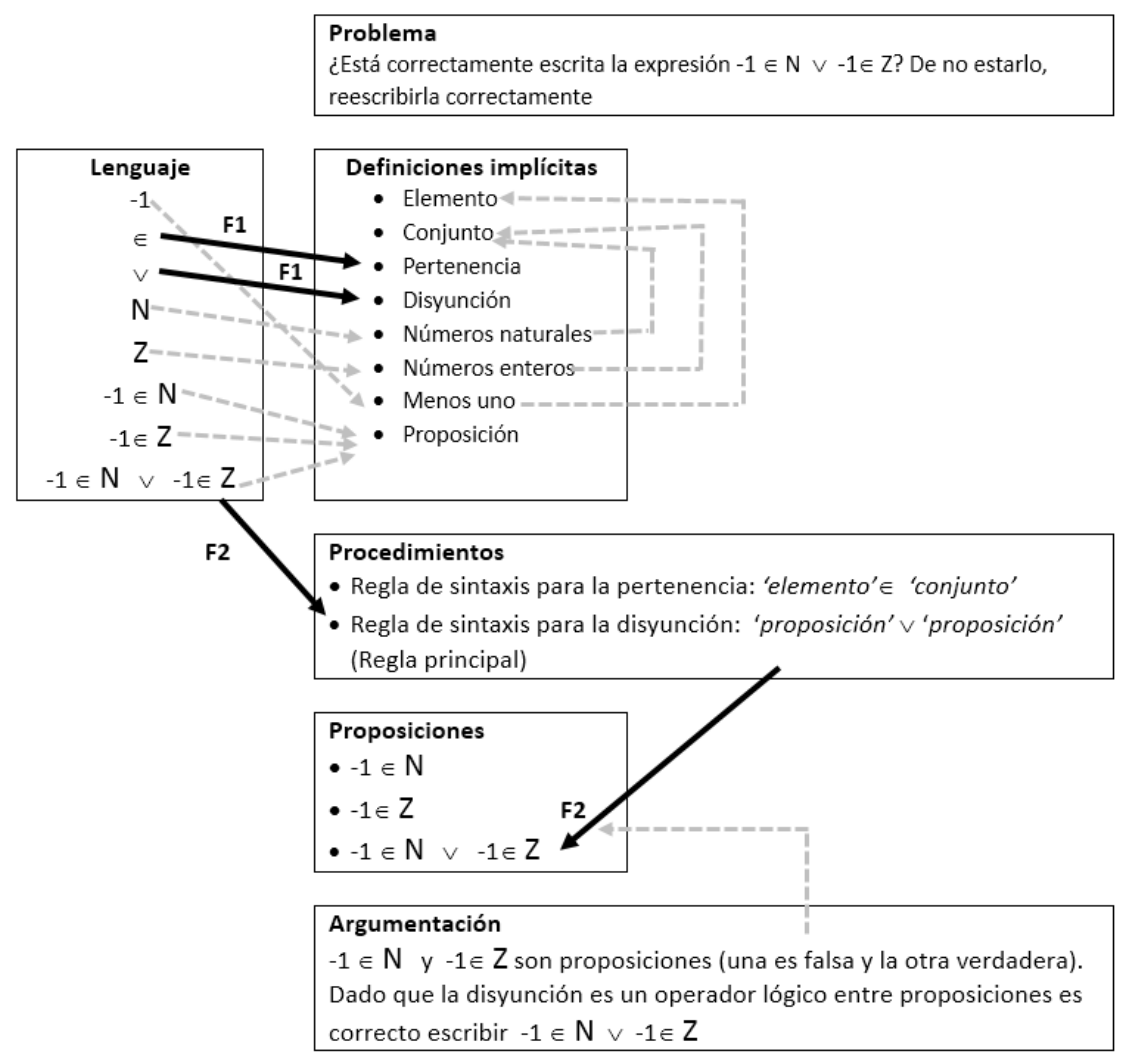

Figura 8. Configuración epistémica y funciones semióticas del análisis de correcta escritura de la expresión $-1 \in \mathbb{N} \vee-1 \in \mathbb{Z}$ 
Sin embargo, 45 estudiantes decidieron que la expresión está incorrectamente escrita y la reescribieron. De esos 45 estudiantes, 24 realizan alguna modificación que está ligada a la proposición $-1 \in \mathrm{N}$ (cambian el -1 por un 1 , o el símbolo ' $\in$ ' por el símbolo ' $\notin$ ', o cambian el conjunto numérico para que el -1 sea un elemento de dicho conjunto). Se manifiesta un conflicto semiótico entre 'estar correctamente escrita' y ser 'verdadera', pero además se evidencia el desconocimiento del valor de verdad de la disyunción.

Otros alumnos (14), además de realizar algunas de las modificaciones mencionadas sobre la expresión $-1 \in \mathrm{N}$, cambian la disyunción por una conjunción, como si no fuera posible la disyunción entre dos proposiciones verdaderas. El hecho de reescribir utilizando dos proposiciones verdaderas, como si esto fuera obligatorio, podría ser una manifestación de no tener establecida la F3 de la disyunción.

\section{Configuración del ítem $-5 \wedge 4 \in \mathbb{R}$}

Los 74 estudiantes que responden que es correcta, no sólo no tienen establecida la F2 del símbolo ' $\wedge$ ' sino que lo usan coloquialmente, como un reemplazo de la conjunción coloquial ' $y$ ', en lugar de entenderlo como un operador lógico. Es decir que es una manifestación de la deficiente construcción del significado del símbolo ' $\wedge$ '.

El error más observado (11 alumnos) fue que lo reescribieran como: $\{-5$, $4\} \in$ R. Esto lleva a formular nuevas preguntas, tales como: ¿esto sería una manifestación de no tener establecida la F2 del símbolo ' $\in$ ' pero sí la del símbolo ' $\wedge$ '?, ¿Podría provenir de una confusión generada por el abuso de notación que se realiza en Matemática con respecto a las convenciones de la Lógica? Esto es, en Matemática es válido escribir -5, $4 \in \mathrm{R}$, mientras que según las reglas de la Lógica debería escribirse: $-5 \in R \wedge 4 \in R$. Las evidencias que se tienen no permiten responder a estos interrogantes, sin embargo resultan de interés para profundizar su estudio en futuras investigaciones. 


\section{Problema}

¿Está correctamente escrita la expresión $-5 \wedge 4 \in R$ ? De no estarlo, reescribirla correctamente
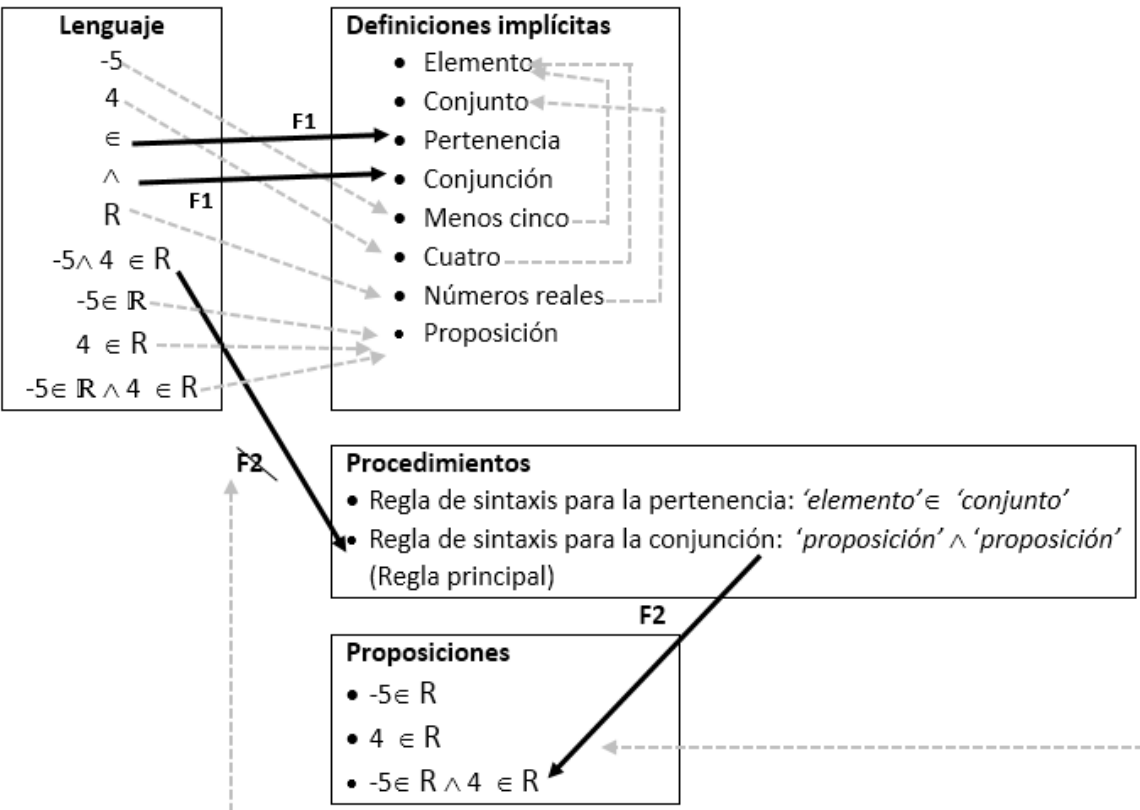

\section{Argumentación}

- '-5' no es una proposición, por lo tanto no es correcto que sea uno de los operandos de una conjunción.

- -5 es un número real, por lo tanto es correcto expresar: $-5 \in R$

- 4 es un número real, por lo tanto es correcto expresar: $4 \in R$

- ' $-5 \in \mathrm{R}^{\prime}$ y ' $4 \in \mathrm{R}^{\prime}$ son proposiciones, por lo tanto pueden ejercer el rol de operandos de una conjunción, con lo cual es correcto

expresar: $-5 \in R \wedge 4 \in R$

Figura 9. Configuración epistémica y funciones semióticas del análisis de correcta escritura de la expresión $-5 \wedge 4 \in \mathbb{R}$ 


\section{Configuración del ítem $4 \in \mathbb{N} \vee \mathbb{Z}$}

El error en la escritura de este ítem guarda cierta similitud con el presentado en el apartado anterior. En relación a esto, parece notable la diferencia en las resoluciones. A este ítem, 35 alumnos lo consideraron bien escrito,

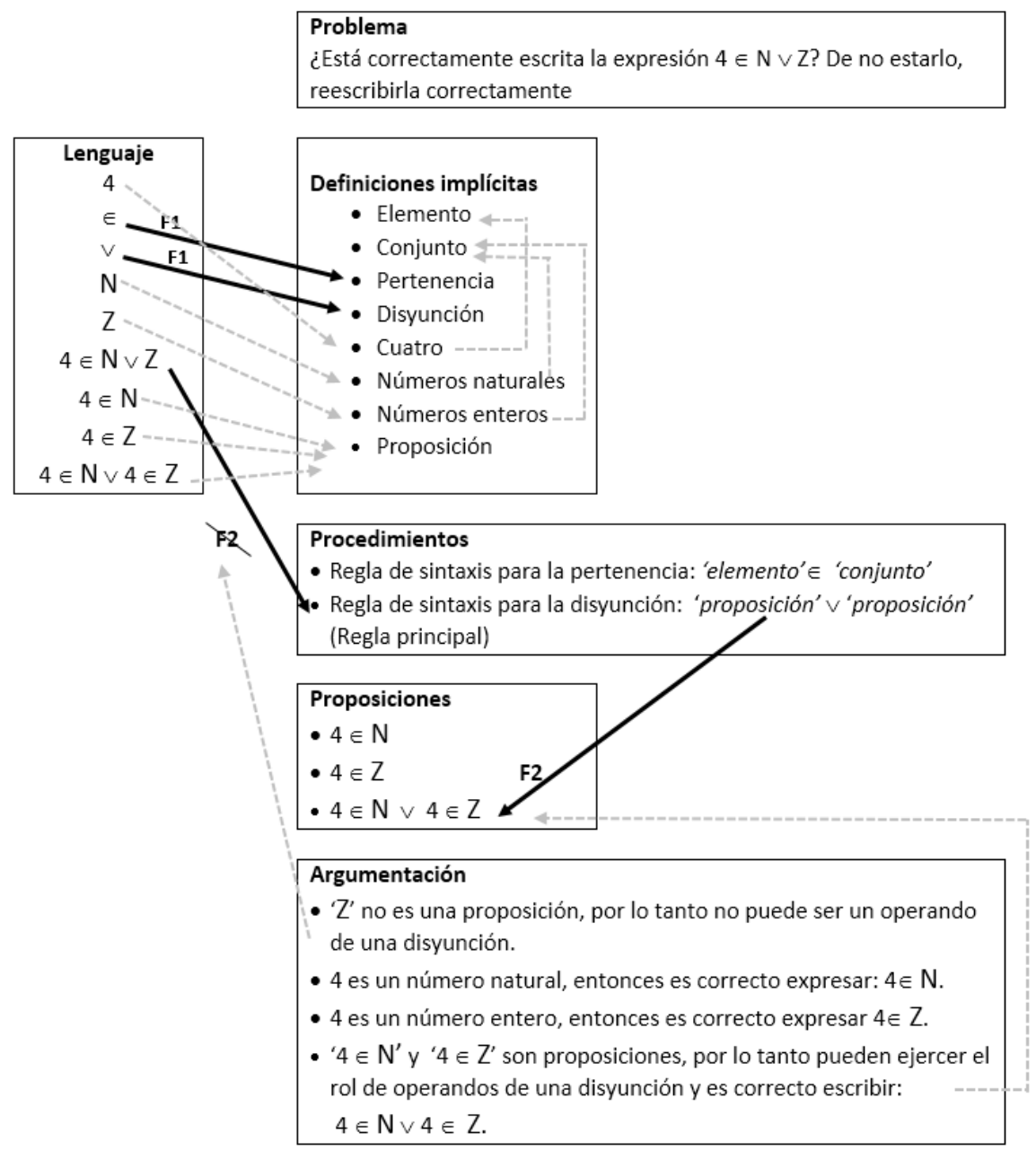

Figura 10. Configuración epistémica y funciones semióticas del análisis de correcta escritura de la expresión $4 \in \mathbb{N} \vee \mathbb{Z}$ 
mientras que en el del apartado anterior $(-5 \wedge 4 \in \mathrm{R})$ fue considerado como correcto por 74 estudiantes. Esto llevaría a pensar que el operador (incorrectamente colocado) entre los conjuntos es reconocido como un error de sintaxis por más alumnos. Sin embargo, de los 45 estudiantes que intentan reescribirlo, 34 alumnos cometen el mismo error: lo reescriben como $4 \in \mathrm{N} \wedge \mathrm{Z}$. es decir que lo que consideran incorrecto no es que las expresiones operadas no son proposiciones, sino que están poniendo en juego (y también de manera incorrecta) el valor de verdad de la disyunción. Estarían considerando el símbolo ' $\mathrm{V}$ ' como excluyente. Esto estaría poniendo de manifiesto que estos estudiantes, por más que hayan reconocido a la expresión como 'Incorrectamente escrita', no tienen construida la F2 del símbolo de disyunción.

Otro error observado (cometido por 6 estudiantes) es haber reescrito la expresión de la siguiente manera: $4 \in \mathrm{N} \wedge 4 \in \mathrm{Z}$. En este caso, manifiestan tener construida la función semiótica relativa a la sintaxis pero, como en el caso anterior, estarían considerando el símbolo ' $\mathrm{V}$ ' como excluyente, y lo cambian por una conjunción probablemente con la intención de darle valor de verdad 'verdadero'. Es decir que en este caso el error de reescritura no está ligado a la sintaxis sino al contenido semántico de la expresión.

\section{Conclusiones}

El objetivo de este trabajo era caracterizar la actividad matemática que realizan alumnos de los primeros cursos universitarios cuando responden tareas en las que ha de usar los símbolos matemáticos: $\in, \subset, \forall, \exists, \wedge, \vee$.

La lectura de una expresión simbólica requiere, en primer lugar, el reconocimiento individual de cada uno de los símbolos, estableciendo su asociación con el lenguaje coloquial. Sin embargo, esta especie de 'traducción uno a uno' no es suficiente para interpretar la expresión y darle sentido a la idea que está expresada. Es necesario establecer el rol que juega cada símbolo interviniente, validarlo a través de argumentaciones y observar que la estructura sintáctica que posee la expresión es correcta en relación con los símbolos que participan en ella.

Por su parte, la escritura de expresiones simbólicas es aún más compleja. La selección de símbolos a utilizar no necesariamente proviene de una 'traducción uno a uno'. En el lenguaje coloquial, una misma idea puede 
expresarse a través de diferentes vocablos que en forma simbólica tienen una única manera de representarse. La escritura simbólica formal en Matemática no admite la ambigüedad del lenguaje coloquial. Por ejemplo, expresiones tales como '2 es un número entero', '2 pertenece al conjunto de los números enteros', '2 es un elemento del conjunto de los números enteros', '2 es un elemento de Z', refieren todas a la misma expresión simbólica ' $2 \in Z$ '. Además, para escribir una expresión simbólica se debe conocer la precisión de la sintaxis que corresponde a cada uno de los símbolos a utilizar, para establecer el orden en que deben escribirse dichos símbolos. Esto requiere, como en el caso de la lectura, reconocer el rol que cada símbolo juega en la expresión y su validación a través de la argumentación. Finalmente, se debe interpretar y dar sentido a la expresión escrita para corroborar su concordancia con la idea que se pretende expresar.

Con relación al objetivo planteado, el análisis realizado pone de manifiesto que, incluso en tareas que se pueden considerar relativamente simples, es necesario considerar una configuración de objetos primarios intervinientes y unas funciones semióticas que establecen una relación de significación entre estos objetos primarios. Por otra parte, la presencia (o ausencia) de estas funciones semióticas se puede considerar como la causa de la respuesta correcta (o incorrecta) de los sujetos participantes.

En la resolución de estos ejercicios, las funciones semióticas definidas (F1, F2 y F3) vinculan distintos objetos primarios en las configuraciones: el Lenguaje con las Definiciones y con los Procedimientos y también los Procedimientos con las Proposiciones. Observando la estructura general de una configuración, puede observase que estas funciones semióticas vinculan Lenguaje y Reglas. El análisis realizado conjugando las funciones semióticas y las configuraciones pone de manifiesto las relaciones que se expresan en la Figura 1, mostrando de qué manera el Lenguaje 'Expresa y soporta' a las Reglas y la forma en que las Reglas 'Regulan el uso' del Lenguaje. También se observa la asociación que se establece entre los objetos que constituyen las Reglas y la incidencia de las Argumentaciones sobre la manifestación de las funciones semióticas F2 ligadas a la sintaxis.

En las tareas de lectura, la vinculación se establece fundamentalmente entre el Lenguaje y los Procedimientos, a través de la F2 correspondiente. En las tareas de escritura, la asociación está dada entre los Procedimientos y 
las Proposiciones. Las Argumentaciones requeridas en cada caso permiten analizar el origen de los errores cometidos.

El hecho de incluir en las configuraciones algunas de las funciones semióticas auxiliares involucradas en la resolución de la actividad (que no son las que están en estudio sino otras que deben establecerse previamente), permite reconocer aún más la complejidad que cada ejercicio tiene y plantea una especie de 'mapa' de las vinculaciones necesarias en cada expresión. Es decir, queda expuesta la trama de funciones semióticas que se pone en funcionamiento al momento de establecer el significado de expresiones simbólicas.

La multiplicidad de procesos involucrados en las tareas de lectura y escritura de expresiones simbólicas, como así también la trama de vinculaciones necesarias para otorgar significado, ponen en evidencia la complejidad cognitiva que la manipulación de símbolos matemáticos posee.

\section{Referencias}

Alcalá, M., (2002). La construcción del lenguaje matemático. Barcelona: Grao.

Arcavi, A. (1994). Symbol sense: informal sense-making in formal mathematics. For the Learning of Mathematics, 14(3), 24-35.

Arcavi, A. (2007). El desarrollo y el uso del sentido de los símbolos. Uno. Revista de didáctica de las matemáticas, 44, 59-75.

Booth, L. (1988). Children's difficulties in beginning algebra. En A.F. Coxford \& A.P. Shulte (Eds.), The Ideas of Algebra, K-12. 1988 Yearbook. Reston, VA: The National Council of Teachers of Mathematics.

Camós, C. and Rodríguez, M. (2009). Exploración del uso de los lenguajes natural y simbólico en la enseñanza de Matemática superior.

Memorias del VI Congreso Iberoamericano de Educación Matemática (VI Cibem). Chile. Retrieved from: http://ebookbrowse.com/articulo-camos-rodriguez-texto-completopdf-d36067393.

Colombano, V., Formica, A. and Camós, C. (2012). Enfoque cognitivista. En M.D. Pochulu, M. y M. Rodríguez (compiladores). Educación Matemática. Aportes a la formación docente desde distintos enfoques 
teóricos (pp. 115-152). Editorial Universitaria de Villa María: Villa María.

Distéfano M. L., Urquijo, S. and González, S. (2010) Una intervención educativa para la enseñanza del lenguaje simbólico. Unión, Revista Iberoamericana de Educación Matemática, 23, 59-71.

Distéfano, M.L.; Aznar, M.A. and Pochulu, M. (2014). Procesos cognitivos y significación de símbolos algebraicos en estudiantes universitarios. Memorias del Congreso Iberoamericano de Ciencia, Tecnología, Innovación y Educación. Buenos Aires. Retrieved from:

http://www.oei.es/congreso2014/contenedor.php?ref=memorias\#16

Duval, R. (2004). Semiosis y pensamiento humano. Colombia: Universidad del Valle. Instituto de educación y pedagogía.

Font, V. (2001). Algunos puntos de vista sobre las representaciones en didáctica de las matemáticas. Philosophy of Mathematics Education Journal, 14, 1-35.

Font, V. and Godino, J. D. (2006). La noción de configuración epistémica como herramienta de análisis de textos matemáticos: su uso en la formación de profesores. Educaçao Matemática Pesquisa, 8 (1), 6798.

Font, V., Godino, J. D. and Gallardo, J. (2013). The emergence of objects from mathematical practices. Educational Studies in Mathematics, 82, 97-124. doi: 10.1007/s10649-012-9411-0

Godino, J. D. (2002). Un enfoque ontológico y semiótico de la cognición matemática. Recherches en Didáctique des Mathematiques, 22 (2/3), 237-284.

Godino, J. D., Batanero, C. and Font, V. (2007). The onto-semiotic approach to research in mathematics education. ZDM. The International Journal on Mathematics Education, 39(1), 127-135. doi: 10.1007/s11858-006-0004-1

Godino, J. D., Bencomo, D., Font, V. and Wilhelmi, M. R. (2007). Pauta de análisis y valoración de la idoneidad didáctica de procesos de enseñanza y aprendizaje de las matemáticas. Departamento de Didáctica de la Matemática. Universidad de Granada. Retrieved from: http://www.ugr.es/ jgodino/funciones-semioticas/pauta_ valoracion_idoneidad_5enero07.pdf 
Godino, J. D., Batanero, C. and Font, V. (2008). Un enfoque ontosemiótico del conocimiento y la instrucción matemática. Acta Scientiae. Revista de Ensino de Ciências e Matemática, 10, 7-37.

Godino, J. D. and Font, V. (2010). The theory of representations as viewed from the onto-semiotic approach to mathematics education. Mediterranean Journal for Research in Mathematics Education, 9(1), 189-210.

Goldin, G. y Stheingold, (2001). System of representations and the development of mathematical concepts. En A. Cuoco y F. R. Curcio (Eds.), The roles of representation in school mathematics (pp. 123). Yearbook 2001. Reston, VA: NCTM.

Gómez Granel, C. (1989). La adquisición del lenguaje matemático: un difícil equilibrio entre el rigor y la significación. Comunicación, lenguaje y educación, 3-4, 5-16.

Hiebert, J. (1988). A theory of developing competence with written mathematical symbols. Educational Studies y Mathematics, (19), 333-355. doi: 10.1007/BF00312451

Kaput, J. (1991). Notations and representations as mediators of constructive processes. En E. von Glasersfeld (Ed.), Radical Constructivism in Mathematics Education (pp. 53-74). Dordrecht, The Netherlands: Kluwer Academic Publishers. Retrieved from: http://www.springerlink.com/content/q6n3h0g5vj122068/fulltext.pdf. Kieran, C. and Filloy Yague, E. (1989) El aprendizaje del álgebra escolar desde una perspectiva psicológica. Enseñanza de las Ciencias, 7(3), 229- 240.

Lacués Apud, E. (2011). Enseñanza y aprendizaje de los sistemas matemáticos de símbolos. Didac, 55-56, 29-35.

Lincoln, Y. and Guba, E. (1985). Naturalistic inquiry. Newbury Park: SAGE Publication, Inc.

Molina González, M. (2007). Desarrollo del pensamiento relacional y comprensión del signo igual por alumnos de tercero de educación primaria. Tesis doctoral. Granada: Universidad de Granada. Retrieved from: http://documat.unirioja.es/servlet/tesis?codigo=1210. Palarea Medina, M. (1998). La adquisición del Lenguaje Algebraico y la detección de errores comunes cometidos en Álgebra por alumnos de 12 a 14 años. Tesis Doctoral. Universidad de La Laguna. Retrieved from: ftp://tesis.bbtk.ull.es/ccppytec/cp90.pdf . 
Palencia, A. and Talavera, R. (2004). Estrategias innovadoras para la comprensión del lenguaje matemático. Revista ciencias de la educación, 1(23), 47-60

Pimm, D. (1990). El lenguaje matemático en el aula. Madrid: Morata.

Rojano, T. (1994). La matemática escolar como lenguaje. Nuevas perspectivas de investigación y enseñanza. Enseñanza de las Ciencias, 12 (1), 45-56.

Rondero, C.; Font, V. (2015). Articulación de la complejidad matemática de la media aritmética. Enseñanza de las Ciencias, 33 (2), 29-49.

Socas, M., and Palarea, M. (1997). Las fuentes de significado, los sistemas de representación y errores en el álgebra escolar. Uno. Revista de Didáctica de las Matemáticas,14, 7-24.

Socas, M. (2007). Dificultades y errores en el aprendizaje de las matemáticas. Análisis desde el enfoque lógico semiótico. Investigación en educación matemática XI, 19-52.

María Laura Distéfano es docente e investigadora de la Facultad de Ingeniería, de la Universidad de Mar del Plata, Argentina.

Marcel David Pochulu es profesor titular del Instituto Académico Pedagógico de Ciencias Básicas y Aplicadas, de la Universidad Nacional de Villa María, Argentina.

Vicenç Font es profesor titular del Departamento de Didáctica de las Ciencias Experimentales y la Matemática, de la Universidad de Barcelona.

Contact Address: La correspondencia relacionada con este artículo se debe enviar al autor. Dirección Postal: Castelli $30333^{\circ} \mathrm{A}$, Mar del Plata, Argentina, C.P.7600. Email: mldistefano@fi.mdp.edu.ar 


\section{Anexo 1 Protocolo del Instrumento Diseñado}

(1 Completar:

\begin{tabular}{|c|c|c|}
\hline Símbolo & ¿Cómo se lee? & $\begin{array}{l}\text { Escriba una expresión utilizando el } \\
\text { símbolo de la que se pueda afirmar que } \\
\text { es VERDADERA }\end{array}$ \\
\hline$\in$ & & \\
\hline$\subset$ & & \\
\hline$\forall$ & & \\
\hline$\exists$ & & \\
\hline$\wedge$ & & \\
\hline$\vee$ & & \\
\hline
\end{tabular}


232 Distéfano, Pochulu and Font-Expresiones simbólicas

2 Determinar si las siguientes expresiones ESTÁN BIEN ESCRITAS. En caso de no estarlo re-escribirlas en forma correcta.

\begin{tabular}{l|l|l}
\hline Expresión & \multicolumn{1}{l}{$\begin{array}{l}\text { Si es la expresión está } \\
\text { BIEN ESCRITA señale } \\
\text { con una x en esta } \\
\text { columna }\end{array}$} & $\begin{array}{l}\text { Si la expresión está } \\
\text { MAL ESCRITA, } \\
\text { re-escribirla en forma } \\
\text { correcta en esta } \\
\text { columna }\end{array}$ \\
\hline$-2 \in \mathbb{Z}$ & & \\
\hline $3 \subset \mathbb{Z}$ & & \\
\hline$\{1 ; 2\} \subset \mathbb{N}$ & & \\
\hline $\mathbb{N} \in \mathbb{Z}$ & & \\
\hline$[2 ; 5] \subset \mathbb{R}$ & & \\
\hline $4 \in \mathbb{N} \wedge-3<0$ & & \\
\hline$-1 \in \mathbb{N} \vee-1 \in \mathbb{Z}$ & & \\
\hline$-5 \wedge 4 \in \mathbb{R}$ & & \\
\hline $4 \in \mathbb{N} \vee \mathbb{Z}$ & & \\
\hline$\forall \mathbb{N} \mathbb{N}>0$ & & \\
\hline$\forall x \in \mathbb{R} \quad \mathrm{x}^{2}>0$ & & \\
\hline$\exists \mathrm{x} \in \mathbb{R} / \mathrm{y}+2=5$ & & \\
\hline$\exists \mathrm{x} \in \mathbb{Z} / \mathrm{x}<0$ & & \\
\hline
\end{tabular}


3 Escribir las siguientes expresiones en lenguaje coloquial o simbólico, según corresponda. Además indicar si son verdaderas o falsas.

\begin{tabular}{l|l|l}
\hline \multicolumn{1}{c}{$\begin{array}{c}\text { En Lenguaje } \\
\text { Coloquial }\end{array}$} & \multicolumn{1}{c}{ En Lenguaje Simbólico } & \\
\hline & $-2,5 \in \mathbb{Z} \vee-1 \in \mathbb{Z} \wedge-1 \in \mathbb{N}$ & \\
\hline & $\forall \mathrm{x}(\mathrm{x} \in \mathbb{Z} \Rightarrow \mathrm{x}<0)$ & \\
\hline & $\exists \mathrm{x}(\mathrm{x} \in \mathbb{Z} \wedge \mathrm{x}<0)$ & \\
\hline & $\exists \mathrm{x} \in \mathbb{N} / \mathrm{x}<0$ & \\
\hline & $\forall \mathrm{x} \in \mathbb{N} \mathrm{x}>0$ & \\
\hline 3 Es un Número & $\forall \mathrm{x} \in \mathbb{N} \mathrm{x}=2 . \mathrm{k} \vee \mathrm{x}=2 . \mathrm{k}+1, \mathrm{k} \in \mathbb{N}$ & \\
Entero e Impar & & \\
\hline $\begin{array}{l}\text { 3 y 5 son números } \\
\text { naturales }\end{array}$ & & \\
\hline $\begin{array}{l}\text { 4 es un número } \\
\text { natural o es un } \\
\text { número entero }\end{array}$ & & \\
\hline $\begin{array}{l}\text { Cada número } \\
\text { entero es menor } \\
\text { que su sucesor }\end{array}$ & & \\
\hline $\begin{array}{l}\text { Algunos números } \\
\text { naturales son } \\
\text { negativos }\end{array}$ & & \\
\hline $\begin{array}{l}\text { El cuadrado de } \\
\text { cualquier número } \\
\text { real es positivo }\end{array}$ & & \\
\hline
\end{tabular}

